\title{
An Oral Tactile Interface for Blind Navigation
}

\author{
Hui Tang, Member, IEEE, and David J. Beebe, Member, IEEE
}

\begin{abstract}
An oral tactile interface was designed and evaluated to provide directional cues through the tactile channel, which may be utilized by a blind traveler to obtain directional guidance in outdoor navigation. The device was implemented as a mouthpiece with a microfabricated electrotactile display on top for tactile presentation onto the roof of the mouth and a tongue touch keypad at the bottom for simultaneous operation by the tongue. An experimental system allowed a user to communicate with a computer tactilely by using the oral interface. Directional cues were presented to the user as line or arrow patterns with four moving directions (leftward, rightward, forward, and backward). Electrotactile presentation on the roof of the mouth was evaluated in experiments of threshold measurement and identification of directional cues. Experimental results from six human subjects showed that the roof of the mouth required stimulation intensities around $15 \mathrm{~V}$ for threshold sensation, and around 25-30 V for comfortable and well-perceived stimulation. Furthermore, identification of leftward or rightward movements was highly accurate while performance on forward or backward moving patterns was mixed and varied considerably among subjects.
\end{abstract}

Index Terms-Blind navigation, electrotactile stimulation, oral tactile interface, tactile communication, tactile display.

\section{INTRODUCTION}

$\mathbf{T}$ RAVELING outdoor can be very challenging for blind individuals. If capable of outdoor navigation, however, a blind individual gains mobility and will be willing to travel outdoors more often leading to improved quality of life [1]. One scenario during blind navigation is how to find the way to a destination (e.g., home) when situated in an unfamiliar location. The blind traveler may find it difficult to know which direction to go and to keep track of that direction [1], [2]. Sighted persons can have similar problems in scenarios equivalent to blind navigation, such as scuba diving under the sea and dismounted soldiers patrolling in an unfamiliar region, where no visual reference is available. If directional cues are available for guidance during blind travel, a blind individual may be able to continuously adjust the traveling path until reaching the final destination with minimal worry of getting lost.

A personal navigation system may assist blind travelers in outdoor navigation by taking advantage of the global positioning system (GPS) [1]-[4]. Such a system typically

Manuscript received March 21, 2001; revised November 7, 2004 and August 20, 2005; accepted September 7, 2005. This work was supported in part by the Army Research Laboratory, in part by the Beckman Institute for Advanced Science and Technology at the University of Illinois at Urbana-Champaign, and in part by the Office of Naval Research.

$\mathrm{H}$. Tang is with the Department of Electrical and Computer Engineering, University of Missouri-Columbia, Columbia, MO 65211 USA (e-mail: tanghu@missouri.edu).

D. Beebe is with the Department of Biomedical Engineering, University of Wisconsin-Madison, Madison, WI 53706 USA (e-mail: dbeebe@engr.wisc.edu).

Digital Object Identifier 10.1109/TNSRE.2005.862696 incorporates a GPS receiver that provides coordinates of the current location and a digital map that allows retrieval of geospatical information about the location. The geospatial information is typically in the form of synthesized sound or speech, which has also been utilized in other assistive navigational aides for obstacle avoidance [5], [6]. A potential problem with voice output is blockage of ambient sound, which is equally important to a blind traveler. In this paper, we explore an alternative means of providing guidance through the tactile channel. Utilization of the tactile channel frees auditory attention to other important tasks (e.g., listening to the sound of coming cars) and reduces the information overload in a particular sensory channel. In addition, tactile presentation can be more intuitive. A comparison among different interfaces using sound/speech and tapping showed that the tapping interface was actually preferred because it was hidden and gave intuitive guidance in a noisy environment [7].

To send information to a person via the tactile channel, tactile stimulation patterns are usually presented onto the skin of the human body with a physical interface called tactile display. In the past, research has been conducted on various tactile displays for the purpose of vision substitution [8], [9] and sensory feedback [10]. Tactile displays may also be used to convert voice or sounds, such as a doorbell or a telephone call, into tactile messages for persons with auditory impairments [11]. The performance of tactile displays can be remarkably dependent on the stimulation method (vibrotactile or electrotactile [12]) and the sensory characteristics of the receptive body regions. Some tactile displays, such as the Optacon, were designed for the fingertip, while other tactile displays such as Tactile Television Substitution Systems targeted body regions of relatively larger area, such as the back and the abdomen [13], [14]. Body regions with larger surface area permit fairly large tactile displays, but these regions possess much lower tactile sensitivity compared with the fingertip. Sensitivity may also vary substantially depending on the stimulation mechanism utilized. The fingertip was shown to be highly sensitive to vibrotactile stimulation, but not to electrotactile stimulation due to its high electrical resistance [12].

This paper presents an oral tactile interface for blind navigation, with an experimental evaluation on the use of oral structures for presenting tactile directional cues. The oral cavity is a very sensitive site compared with most other body locations. Oral structures have a cortical mapping similar in size to that of the hands, while the entire trunk and lower part of the body have a relatively small mapping in somatic sensory cortex [15]. Psychophysical studies on force sensitivity and two-point discrimination showed that some oral structures, such as the tongue tip, were more sensitive than the fingertip [16], [17]. A study testing the tongue perception of electrotactile stimulation with 


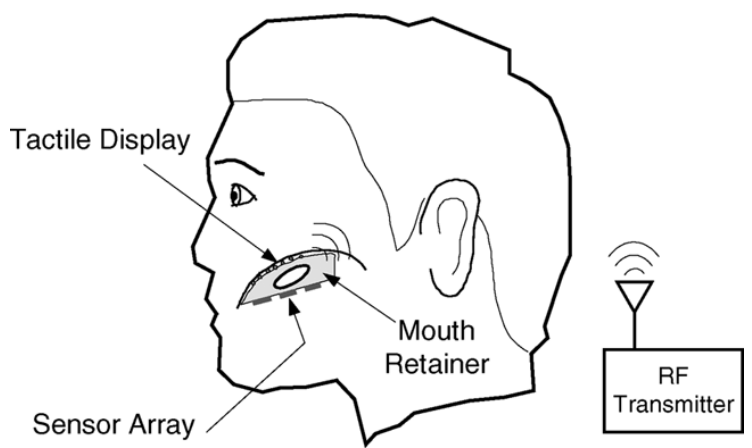

Fig. 1. Oral tactile interface is envisioned as a retainer-like device with both input and output capabilities. Interface communicates with the outside via wireless communication.

a 49-point stimulator array indicated that the tongue required a voltage of 5-15 $\mathrm{V}$ for electrotactile stimulation, which was $1 \%-3 \%$ of the voltage required for stimulating the fingertip [18].

A few tongue-operated devices have been explored for use inside the oral cavity, such as a palatometer for electropalatography [19], IBM's Tonguepoint [20], NewAbilities' tongue touch keypad [21], and a tongue mouse [22]. These devices detect the pressure or force from the tongue, and thus may be utilized to send out message through silent and hands-free operation. In this study, an oral tactile interface capable of two-way communication was designed, which employed a flexible oral electrotactile display for providing directional cues and a tongue touch keypad for sending out feedback or confirmation. In particular, electrotactile presentation on the roof of the mouth and performance on identifying tactile directional cues were further studied in human subject experiments.

\section{ORAL TACTILE INTERFACE}

\section{A. Overall Design}

The oral tactile interface for navigational guidance is envisioned as a wearable device that can be worn inside the mouth like a dental retainer. While the actual size of a retainer may vary from one individual user to another, the dimensions of the device are approximately $3 \mathrm{~cm}$ long and $2 \mathrm{~cm}$ wide. As shown in the conceptual block diagram in Fig. 1, the retainer-like oral tactile interface will be embedded with a tactile display, a sensor array, and additional electronic components for control and wireless communication. The tactile display contains an array of stimulators that presents dynamically refreshable tactile patterns onto oral structures. The user perceives and recognizes the patterns and retrieves guidance information conveyed in these patterns. The sensor array consists of a number of pressure-sensing elements that allow a user to enter commands and controls with the tongue tip. Eventually, the mouthpiece will be powered by a small battery and communicate with a pocket transceiver outside the oral cavity via radio frequency (RF) transmission.

For experimental purposes, a prototype of the oral tactile interface was designed with an electrotactile display for the roof of the mouth and a sensor array for the tongue, as shown in Fig. 2. This configuration allowed constant contact between the tactile display and the oral surface while permitting simultaneous operation on the sensor array by the tongue. The basic supporting

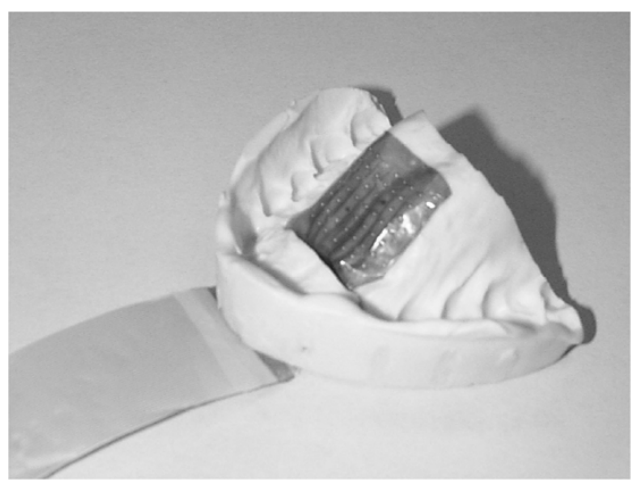

(a)

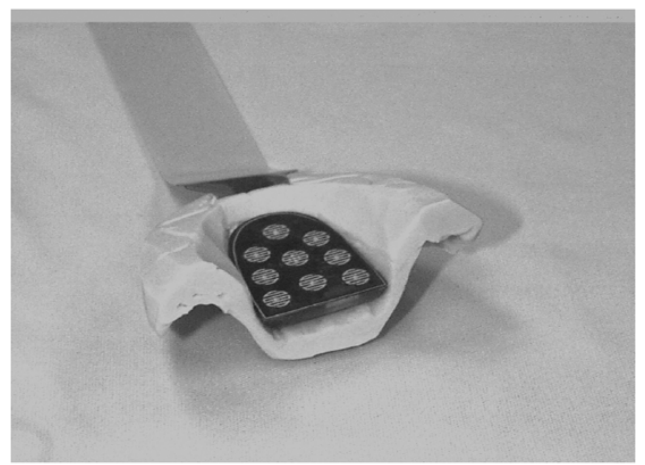

(b)

Fig. 2. Prototype of the oral tactile interface designed as a mouthpiece that fits the upper teeth, with (a) a flexible $7 \times 7$ tactor array on top, and (b) tongue touch keypad at the bottom. In figure (a), the heat seal connector cable exits from underneath the mouth impression via a narrow opening in the impression.

structure of the prototype was a mouth impression that could be custom-made for individual subjects using silicone impression material (CutterSil Putty Plus, Heraeus Kulzer, Inc., South Bend, IN). The mouth impression was relatively bigger than a dental retainer since it was fitted to the roof of the mouth as well as the upper teeth. A thin-sheet flexible oral tactile display with an array of $7 \times 7$ stimulators was attached on top of the impression using silicone adhesive, with electrotactile stimulators facing upwards. The sensor array located at the bottom of the mouth impression was the Tongue Touch Keypad (TTK), a commercial product made by NewAbilities (Mountain View, CA), which communicated with the outside through wireless transmission.

\section{B. Flexible Oral Electrotactile Display}

A polyimide-based flexible oral electrotactile display with $7 \times 7$ dome-shaped tactors was designed to present electrotactile patterns onto the roof of the mouth. The display, as shown in Fig. 3, was fabricated with thin-film and electroplating processes [23]. The overall dimensions of the tactor array are $18.5 \times 18.5 \mathrm{~mm}^{2}$, with each tactor about $200 \mu \mathrm{m}$ in height and $700 \mu \mathrm{m}$ in diameter. The center-to-center spacing between adjacent tactors is $2.54 \mathrm{~mm}$, approximating the two-point resolution of the fingertip, which was measured to be $2.09 \mathrm{~mm}$ with a standard deviation of $0.57 \mathrm{~mm}$ [24]. The flexibility of the display makes it possible to attach the oral display to the roof of the mouth with good contact. The dome-shaped geometry 


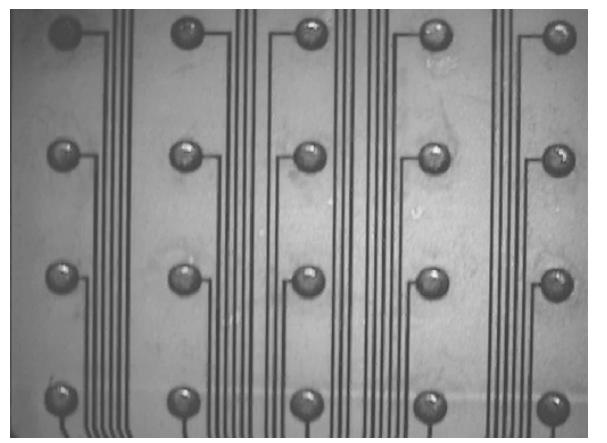

Fig. 3. Oral electrotactile display is a stimulator array fabricated on a flexible polyimide support. Dome-shaped stimulators were electroplated with nickel, $700 \mu \mathrm{m}$ in diameter and $200 \mu \mathrm{m}$ in height.

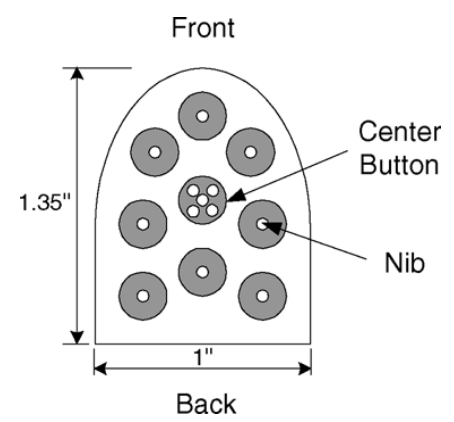

Fig. 4. Layout of buttons on the tongue touch keypad. Each flat circular button is around $3 \mathrm{~mm}$ in diameter. Nibs (raised dots) on the buttons provide tactile cues for tongue exploration.

of the tactors provides more uniform current distribution and a larger surface area for electrotactile stimulation, which may improve the dynamic range of the stimulation current and the quality of the tactile sensation by reducing the localized current density.

\section{TTK}

The tongue touch keypad is a tongue-operated device originally designed for disabled individuals to control electric wheelchairs, electric beds, computers, etc. The acrylic package of a TTK is embedded with control electronics, as well a RF transmitter for wireless communication to the outside. As shown in Fig. 4, the bottom of the package is a miniaturized keyboard with nine flat keypads that can respond to light pressure from the tongue. All keypads have small round plastic hemispheric bumps or "nibs" that serve as tactile cues for tongue exploration. When the TTK is utilized to control the mouse pointer on a computer screen, pushing the center button will trigger a mouse click, and pushing the other eight buttons will cause the mouse pointer to move in eight different directions (up, down, left, right, up-left, up-right, down-left, down-right).

\section{EXPERIMENTAL SYSTEM}

An experimental system was built to allow a user to communicate with a computer tactilely by using the interface worn inside the oral cavity. The user received directional guidance from the PC computer and sent back confirmation messages to the computer by pushing buttons on the tongue touch keypad. As illustrated in Fig. 5(a), the system employed a programmable

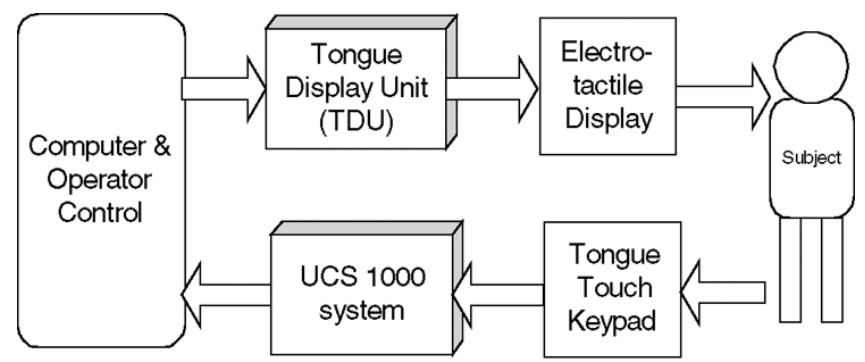

(a)

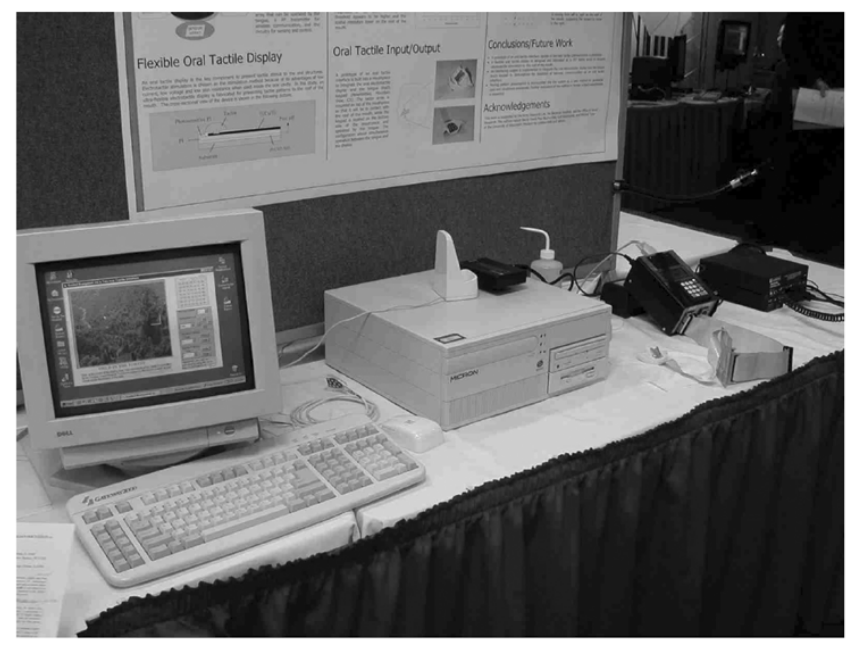

(b)

Fig. 5. Experimental system was implemented to allow communication between the oral tactile interface and a PC computer: (a) block diagram of the system and (b) a photo of the setup that shows from left the computer screen, the infrared receiver, the waveform generator, and the TTK controller.

waveform generator to deliver various electrotactile patterns onto the flexible oral tactile display, based on commands received from the computer through a serial RS-232 link. Signals from the tongue touch keypad were routed to the computer via the UCS 1000 system developed by NewAbilities for the tongue touch keypad. Fig. 5(b) shows a photo of the system configuration.

\section{A. System Apparatus}

The waveform generator delivered stimulating waveforms that could be individually adjusted for up to 144 stimulators. The interconnection between the waveform generator and the electrotactile display consisted of a heat seal connector (Elform, Inc., Reno, NV), a printed circuit board, and two standard 40-pin IDC cables. The flexible heat seal connector (line traces in width of $0.25 \mathrm{~mm}$ and a pitch of $0.5 \mathrm{~mm}$ ) connected the bonding pads of the tactile display to the printed circuit board. The waveform applied to each activated tactor was a capacitively coupled voltage pulse train, as shown in Fig. 6. A set of waveform parameters, similar to what was used for tongue stimulation [18], was adopted for stimulation on the roof of the mouth. The sensations for stimulation at different intensities are described in Section IV-B on sensory thresholds. For safety reasons, the waveform generator operated on an internal rechargeable battery. 


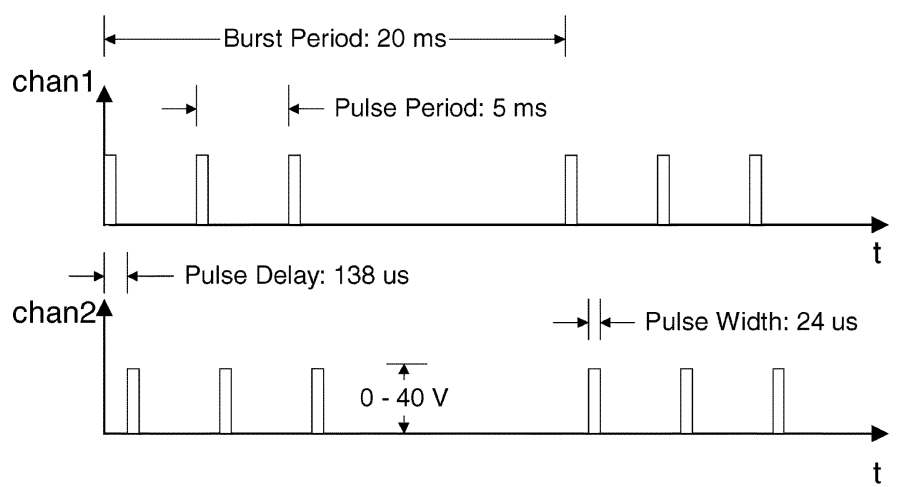

Fig. 6. Stimulation waveforms delivered to two adjacent active tactors. Each waveform is a voltage-clamped monophasic pulse train with three pulses in each burst and $20 \mathrm{~ms}$ between onsets of bursts. Waveforms applied to active tactors are identical except an offset of $138 \mu$ s between adjacent channels.

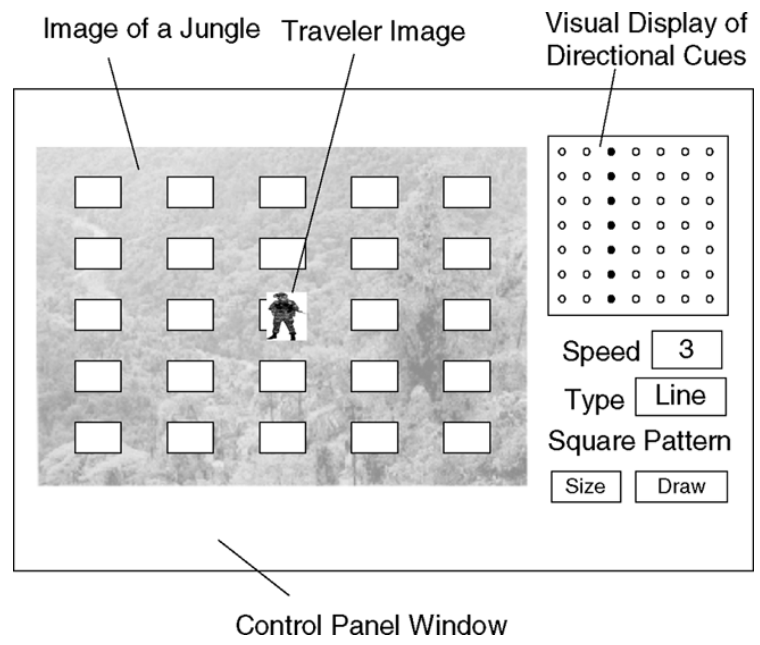

Fig. 7. Control panel of the Visual Basic program that controls the experimental system. Directional cue is generated when the experimenter pushes an arrow key on the computer keyboard (not shown). Program responds to a keystroke on the tongue touch keypad by shifting the traveler image in the corresponding direction. Subject will not see the control panel during experiment.

The UCS 1000 system consisted of a TTK RF receiver, an infrared mouse receiver, a universal controller, and a liquid crystal display (LCD) with an infrared transmitter. The low-frequency $\mathrm{RF}$ signals from the tongue touch keypad were first detected by the TTK receiver, and then processed by the universal controller. Under the control of the universal controller, the infrared transmitter located on top of the interactive menu display sent infrared signals to the infrared mouse receiver, which controlled the mouse pointer on the computer screen.

\section{B. Software}

A Visual Basic program was designed and implemented to allow an operator to send a directional cue to the user by pushing one of the arrow keys on the computer keyboard. A visual version of the directional cue was displayed on the upright indicator array of the control panel, as shown in Fig. 7. The directional cues were implemented as moving lines or arrows to indicate the direction the user should take. The "Type" box on the control panel allowed selection of either a line or arrow pattern. Each line or arrow may move in one of the four direc-

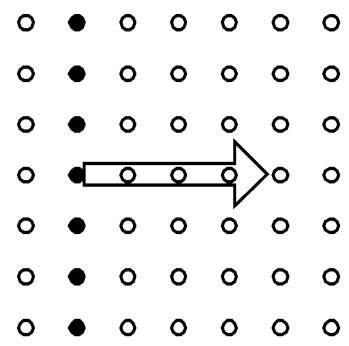

(a)

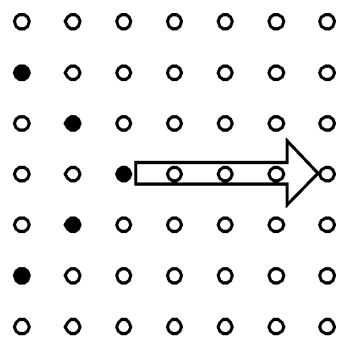

(b)
Fig. 8. Directional cues indicating the direction to the right. (a) Moving-line pattern. (b) Moving-arrow pattern.

tions, i.e., left, right, forward (toward the lips), and backward (toward the throat). An example of a moving-line pattern and a moving-arrow pattern are shown in Fig. 8. For each moving pattern, the line or arrow stayed in the current position for $0.3 \mathrm{~s}$ before shifting one column or one row to the next position. In addition, the program allowed selection of different square patterns utilized in the experiment on sensory thresholds, which is described in Section IV-A.

The control panel also contained a jungle scene on the left with a $5 \times 5$ array of positions to which the image of a traveler can move. After a directional cue was sent, the program detected the movement of the mouse pointer and translated it into a shift of the traveler image on the computer screen, which served as visualization of the confirmation message sent from the user. Since both identification of tactile patterns and operation of the tongue touch keypad could introduce errors, their performance should be evaluated separately. The following section describes how identification of tactile directional cues was evaluated in human subject experiments. Readers may refer to another study [21] regarding the accuracy of using the tongue touch keypad as an input device.

\section{EVALUATION}

Since the roof of the mouth is a site seldom utilized for tactile applications, the intensities required for electrotactile stimulation on this site should be evaluated. Low-intensity stimulation is essential for a wearable device that demands low power consumption. Two experiments were conducted on six human subjects to measure the sensory thresholds and the accuracy of identifying directional cues respectively. For each subject, the experiments were repeated in five sessions, with at least $24 \mathrm{~h}$ between two consecutive sessions. Each session lasted for about $1 \mathrm{~h}$.

The six human subjects, including four males and two females, were recruited from undergraduate and graduate students on campus at the University of Illinois at Urbana-Champaign, with an average age of 27.2 years. Each subject received a modest payment for his/her participation, but the participation was entirely voluntary. Each individual was fitted with a custom-made silicon impression with a separate flexible oral display attached to it.

\section{A. Sensory Thresholds}

In the experiment of threshold measurement, sensory thresholds were obtained by measuring intensities of the electrotac- 


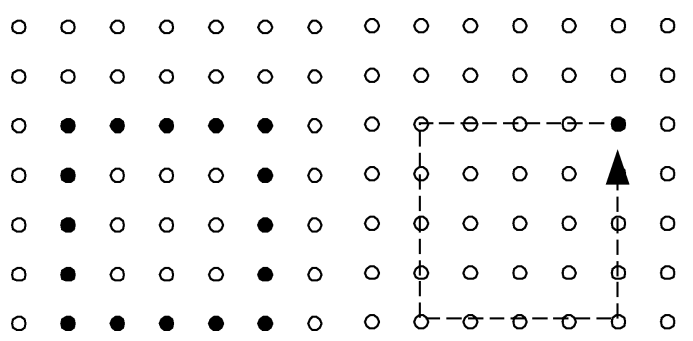

Fig. 9. (a) Static square of size 5 and (b) dynamic square of size 5 "drawn" by activating the dots on the sides of the square sequentially.

tile stimulation just noticeable to human subjects. In general, a higher sensory threshold suggests that it is more difficult for the receptive body region to perceive the stimulation. The test patterns were a static square of size 5 and a dynamic square of size 5, as shown in Fig. 9. A square of size 5 had five dots on each side. For static presentation, all dots on the sides of the square were activated simultaneously. For dynamic presentation, the square was "drawn" by activating the dots on the sides sequentially and counterclockwise, with each dot active for $0.12 \mathrm{~s}$ and no time delay before the activation of the next dot. Similar static patterns of the same size have been utilized for threshold measurement on other tactile displays [25], [26]. Dynamic patterns were included to investigate the effect of different presentation modes on sensory thresholds, i.e., whether the sensitivity would be lower for a stimulus moving on the skin surface.

An adaptive psychophysical approach called two-alternative forced choices (2AFC) was utilized in the experiment to minimize any possible bias from human subjects [27]. This method tracked the sensory threshold through a number of trials, with two intervals in each trial. One interval contained a stimulus (i.e., a square pattern), and the other was null, with duration of $3 \mathrm{~s}$ for each interval. The subject was asked to identify which interval contained the stimulus by pointing at the number " 1 " or " 2 " on a card. Depending on the response from the subject, the intensity of stimulation was adjusted accordingly. The intensity was increased following one incorrect response, and decreased following two consecutive correct responses. For each reversal, the increment or decrement (step size) was half of the previous one. This method tracks the threshold at which the stimulation can be detected with a possibility of $71 \%$. Initially, the intensity was set at a magnitude that was well perceived. Each measurement ended when the step size fell within $1 \%$ of the entire intensity range. The intensity of the stimulation was recorded as the amplitude of the voltage pulse waveform applied.

Threshold values for each subject in dynamic and static presentation modes were plotted in Fig. 10. The threshold values were averaged over five sessions, with the vertical error bars showing the variation of sensory thresholds among sessions. The working-level intensities, at which subjects found the sensation comfortable and well perceived, were shown in the same figure. Low-intensity electrotactile stimulation on the roof of the mouth was perceived as an itching sensation, while stimulation with intensity at the working level produced a sensation described as being touched by a blunt tip. However, a pricking sensation occasionally occurred, possibly because some tactors were closer to dental nerve fibers than others. The pricking sensation tended to disappear for the same intensity of stimulation

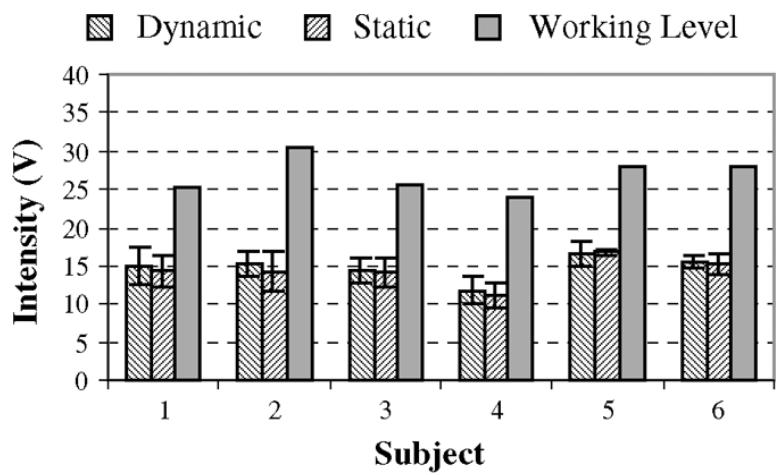

Fig. 10. Average intensities at the threshold level for static and dynamic presentation modes and the intensities at the working level where stimulation was comfortable and well-perceived.

TABLE I

ORGANIZATION OF THE EXPERIMENT ON IDENTIFICATION OF DIRECTIONAL CuEs. EACH SESSION CONTAINS Two PARTS: Two-ChOICE DiRECTIONAL Cues and Four-Choice Directional Cues. PART I Consists of Four SETS OF PATTERNS IN RANDOM ORDER AND PART II CONSISTS OF TWO SETS OF Patterns, With Three Blocks in EACH SeT

\begin{tabular}{ccccc}
\hline & Set 1 & Set 2 & Set 3 & Set 4 \\
\hline $\begin{array}{c}\text { Part I: } \\
\text { Two- } \\
\text { choice }\end{array}$ & $\begin{array}{c}\text { 6 left or right- } \\
\text { moving line } \\
\text { patterns }\end{array}$ & $\begin{array}{c}\text { 6 forward or } \\
\text { backward- } \\
\text { moving line } \\
\text { patterns }\end{array}$ & $\begin{array}{c}\text { 6 left or right- } \\
\text { moving } \\
\text { arrow } \\
\text { patterns }\end{array}$ & $\begin{array}{c}\text { 6 forward or } \\
\text { backward- } \\
\text { moving arrow } \\
\text { patterns }\end{array}$ \\
\hline $\begin{array}{c}\text { Part II: } \\
\text { Four- } \\
\text { choice }\end{array}$ & $\begin{array}{c}\text { 3 blocks, with } \\
\text { 4 line patterns } \\
\text { (left, right, } \\
\text { forward, or } \\
\text { backward) } \\
\text { each block }\end{array}$ & $\begin{array}{c}\text { 3 blocks, with 4 } \\
\text { arrow patterns } \\
\text { (left, right, } \\
\text { forward, or } \\
\text { backward) each } \\
\text { block }\end{array}$ & \\
\hline
\end{tabular}

after initial sessions, indicating adaptation of the nerves to the stimulation.

\section{B. Identification of Directional Cues}

In this experiment, tactile directional cues were presented as moving-line or moving-arrow patterns described in Section III-B on System Software. The subjects were asked to identify the direction of the movement, and then pick up an arrow pointing to the same direction on a card. Stimulation at the working-level intensities was used throughout the experiment for all subjects. As summarized in Table I, each session for this experiment contained two parts: one for identification of two-choice directional cues and the other on four-choice directional cues. In Part I on two-choice directional cues, each subject was presented with four consecutive sets of patterns in random order, with six patterns in each set. Respectively, these four sets included six line patterns in either left or right-moving direction, six line patterns in either forward or backward-moving direction, six arrow patterns in either left or right-moving direction, and six arrow patterns in either forward or backward-moving direction. In each set, the same moving direction was repeated three times. In Part II on four-choice directional cues, each subject was given one set of moving-line patterns and one set of moving-arrow patterns. Each set contained three blocks of four patterns in random order, with one pattern for each direction. The starting set was alternated between line patterns and arrow patterns. 


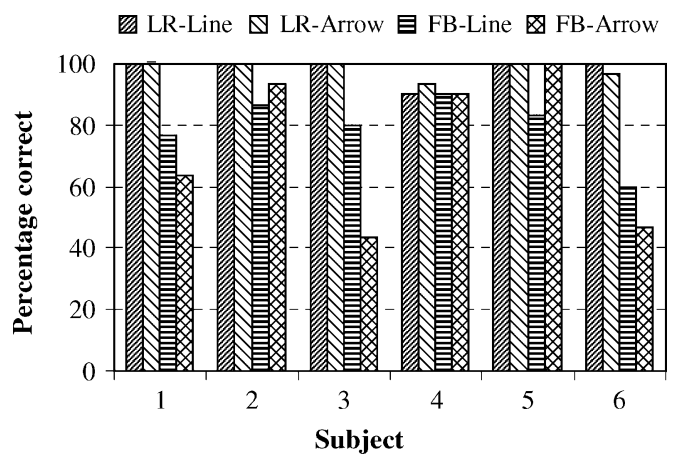

Fig. 11. Percentages of correct responses on identification of two-choice directional cues in four pattern categories.

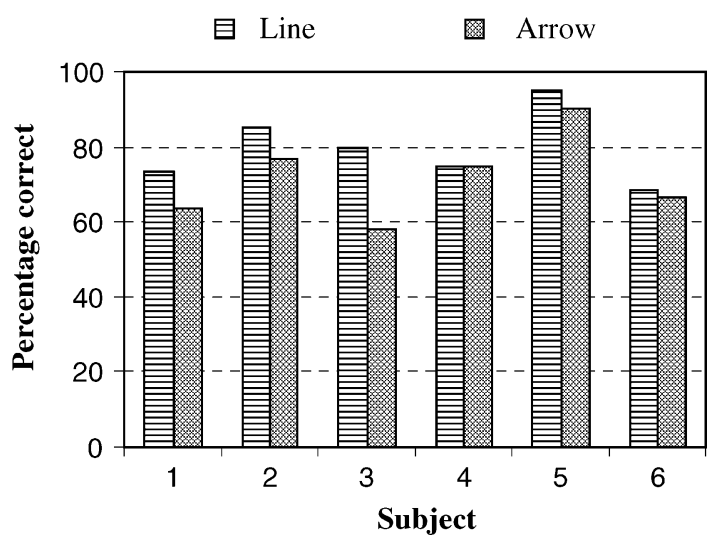

Fig. 12. Percentages of correct responses on identification of four-choice directional cues in two pattern categories.

Subject performance in perceiving and identifying directional cues was summarized as the percentage of correct responses for each subject over the five sessions. The performance with two-choice directional cues for each subject is shown in Fig. 11 (Line: moving line patterns; Arrow: moving arrow patterns; L: moving leftward; R: moving rightward; F: moving forward; B: moving backward). The performance on four-choice directional cues for each subject is shown in Fig. 12. Confusion among four-choice directional cues is summarized in Table II (a) for all line patterns and (b) for all arrow patterns. Each letter in the first column of each confusion matrix indicates the movement presented, and each letter in the first row indicates the movement perceived. Each element in the matrices indicates a percentage of the movements in one direction that were perceived as movements in another direction. Further discussions regarding the experimental results are presented in the following section.

\section{DISCUSSION}

The threshold experiment showed that the roof of the mouth was very sensitive to electrotactile stimulation in terms of the intensities required. Although the thresholds on the roof of the mouth (around 10-20 V) were a little higher than those on the tongue (around 5-15 V), they were much lower than the intensities required for electrotactile stimulation on the fingertip. The intensities at the working level for all subjects were also
TABLE II

CONFusion MATRICES ON Four-ChOICE DiRECTIONAL CuES. EACH LETTER IN THE FIRST COLUMN INDICATES THE MOVING DIRECTION OF THE PATTERNS PRESENTED, AND EACH LETTER IN THE HEADING Row INDICATES THE PERCEIVEd Moving Direction (L: LeFTWARD, R: RightwARd, F: ForWARD, B: BACKWARD). EACH ELEMENT IN THE MATRICES INDICATES A PERCENTAGE of the Presented Movements in One Direction That Were Perceived AS MOVEMENTS IN ANOTHER DIRECTION

(a)

\begin{tabular}{ccccc}
\hline & $\mathrm{L}$ & $\mathrm{R}$ & $\mathrm{F}$ & $\mathrm{B}$ \\
\hline $\mathrm{L}$ & 88.9 & 1.1 & 5.6 & 4.4 \\
$\mathrm{R}$ & 0.0 & 84.4 & 6.7 & 8.9 \\
$\mathrm{~F}$ & 4.4 & 5.6 & 62.2 & 27.8 \\
$\mathrm{~B}$ & 2.2 & 1.1 & 14.4 & 82.2 \\
\hline
\end{tabular}

(b)

\begin{tabular}{ccccc}
\hline & $\mathrm{L}$ & $\mathrm{R}$ & $\mathrm{F}$ & $\mathrm{B}$ \\
\hline $\mathrm{L}$ & 84.4 & 0.0 & 5.6 & 10.0 \\
$\mathrm{R}$ & 1.1 & 80.0 & 5.6 & 13.3 \\
$\mathrm{~F}$ & 0.0 & 4.4 & 63.3 & 32.2 \\
$\mathrm{~B}$ & 12.2 & 3.3 & 25.6 & 58.9 \\
\hline
\end{tabular}

at a low-voltage range, from 25 to $30 \mathrm{~V}$. The experiment indicated that low-voltage stimulation was adequate for the roof of the mouth, an important feature for an oral tactile interface that needs to be further miniaturized into a fully self-contained package.

The experiment on two-choice directional cues showed that subjects could identify the left or right-moving direction with almost perfect performance, regardless of whether the pattern was a moving line or arrow. However, it could be difficult for a subject to identify the forward or backward movement of a pattern, though some subjects had excellent performance in this task as well. The average percentages of correct responses from the six subjects were $77 \%$ for a forward-moving line pattern, $82 \%$ for a backward-moving line pattern, $71 \%$ for a forwardmoving arrow pattern, and $74 \%$ for a backward-moving arrow pattern. Performance on moving-line patterns was slightly better than that on moving-arrow patterns, although ANOVA did not show a significant difference between moving-line and movingarrow patterns.

In the experiment on four-choice directional cues, the overall accuracy decreased when a mixture of laterally and vertically moving patterns were presented. In general, the accuracy on laterally moving patterns was still much higher than that on vertically moving patterns. For a left or right moving pattern, one direction was seldom identified as the opposite direction, no matter whether it was a moving line or a moving arrow. As shown in both matrices in Table II, the confusion between left and right-moving patterns was very low, up to $1 \%$. However, they could be perceived as patterns moving vertically, especially as backward moving patterns. In addition, a forward-moving pattern was more frequently perceived as a backward-moving pattern than a backward-moving pattern was perceived as a forward-moving pattern. For moving arrow patterns, $10 \%$ of leftmoving patterns were identified as moving backward, and $13 \%$ of right-moving patterns were identified as moving backward. Overall, the perception from the roof of the mouth on the left or right movement was more distinctive compared with the perception on the forward and backward movement. 
One may wonder why the roof of the mouth was more capable of distinguishing left and right moving patterns. There could be a couple of hypothetic explanations. 1) Since one side of the somatic sensory cortex received afferent somatic sensory signals from the opposite side of the body, the corresponding excited spot on the somatic cortex switched sides when the stimulus (a line or an arrow) moved leftward or rightward across the central column of the tactor array. This switching may be easier to identify. 2) The sensory nerve fibers on the roof of the mouth may be more vertically aligned, and, therefore, different nerve fibers may be stimulated when a pattern was moving left or right. 3) The two-point resolution in the horizontal direction may be higher than that in the vertical direction. Further evidence is needed to validate any one of these hypotheses.

Even though the subjects showed mixed performance in identification of four-choice directional cues, the finding that left and right moving patterns could be accurately identified as demonstrated in the two-choice experiment suggested the potential of the oral tactile interface for blind navigation. As one example, a basic set of two alternatives with right and left directional cues would be enough for a blind traveler to keep track of the travel direction, as turning around can be indicated by continuously sending the left (or right)-turn signal. Thus, the interface could be useful in assisting the blind traveler in outdoor navigation while freeing auditory attention to environmental sound.

\section{CONCLUSION}

The design, implementation, and evaluation of an oral tactile interface for blind navigation have been presented. The oral tactile interface was implemented as a silicone-based mouthpiece with an oral electrotactile display for providing tactile cues to the roof of the mouth and a tongue touch keypad for sending out feedback with the tongue. The roof of the mouth was chosen for tactile presentation because the tactile display could be in constant contact with the oral surface while allowing simultaneous tongue operation on the keypad. An experimental system was built to enable two-way tactile communication between a computer and a user wearing the oral tactile interface. Psychophysical evaluation in human subject experiments showed that the roof of the mouth required relative low intensities for electrotactile stimulation, a feature desirable for energy-efficient wearable aids. The experiments also indicated that identification of left or right moving patterns was highly accurate, while errors on forward or backward moving patterns were more frequent and varied considerably among subjects. Nevertheless, the feasibility of using the oral tactile cues for navigational guidance was demonstrated.

\section{ACKNOWLEDGMENT}

The authors would like to thank P. Bach-y-Rita, K. Kaczmarek, and especially M. Tyler at the University of Wisconsin-Madison for advice and providing the waveform generator.

\section{REFERENCES}

[1] R. G. Golledge, J. R. Marston, J. M. Loomis, and R. L. Klatzky, "Stated preferences for components of a personal guidance system for nonvisual navigation," J. Visual Impairment Blindness, pp. 135-147, Mar. 2004.

[2] A. Y. J. Szeto, "A navigational aid for persons with severe visual impairments: A project in progress," presented at the Proc. 25th Annu. Int. Conf. IEEE Eng. Med. Biol. Soc., Cancun, Mexico, Sep. 17-21, 2003.

[3] A. Helal, S. Moore, and B. Ramachandran, "Drishti: An integrated navigation system for visually impaired and disabled," in Proc. 5th Int. Symp. Wearable Computer, Zurich, Switzerland, Oct. 2001, pp. 149-156.

[4] H. Makino, I. Ishii, and M. Nakashizuka, "Development of navigation system for the blind using GPS and mobile phone combination," in Proc. 18th Annu. Int. Conf. IEEE Eng. Med. Biol. Soc., Amsterdam, The Netherlands, 1996, pp. 506-507.

[5] S. Shoval, J. Borenstein, and Y. Koren, "Auditory guidance with the Navbelt-A computerized travel aid for the blind," IEEE Trans. Syst., Man, Cybern. C, Appl. Rev., vol. 28, no. 3, pp. 459-467, Aug. 1998.

[6] S. L. Grow, "The use of the sonic pathfinder as a secondary mobility aid for travel in business environments: A single-subject design," J. Rehabil. Res. Devel., vol. 36, no. 4, pp. 333-340, 1999.

[7] D. A. Ross and B. B. Blasch, "Wearable interfaces for orientation and wayfinding," in Proc. ACM ASSETS, Arlington, VA, Nov. 13-15, 2000, pp. $193-200$.

[8] J. C. Bliss, M. H. Katcher, C. H. Rogers, and R. P. Shepard, "Optical-totactile image conversion for the blind," IEEE Trans. Man-Machine Syst., vol. MMS-11, pp. 58-65, 1970.

[9] C. C. Collins, "Tactile television-Mechanical and electrical image projection," IEEE Trans. Man-Machine Syst., vol. MMS-11, pp. 58-70, 1970.

[10] A. Y. J. Szeto and R. R. Riso, "Sensory feedback using electrical stimulation of the tactile sense," in Rehabilitation Engineering, R. V. Smith and J. H. Leslie, Jr., Eds. Boca Raton, FL: CRC Press, 1990, pp. 29-78.

[11] C. E. Sherrick, "Basic and applied research on tactile aids for deaf people: Progress and prospects," J. Acoust. Soc. Amer., vol. 75, pp. $1325-1342,1984$

[12] K. A. Kaczmarek, J. G. Webster, P. Bach-y-Rita, and W. J. Tompkins, "Electrotactile and vibrotactile displays for sensory substitution systems," IEEE Trans. Biomed. Eng., vol. 38, no. 1, pp. 1-16, Jan. 1991.

[13] P. Bach-y-Rita, "Neurophysiological basis of a tactile vision-substitution system," IEEE Trans. Man-Machine Syst., pp. 108-110, Mar. 1970.

[14] P. Bach-y-Rita, C. C. Collins, F. A Saunders, B. White, and L. Scadden, "Vision substitution by tactile image projection," Nature, vol. 221, pp. 963-964, 1969.

[15] A. C. Guyton, Textbook of Medical Physiology, 8th ed. Philadelphia, PA: W. B. Saunders, 1991.

[16] R. C. Grossman, B. F. Hattis, and R. L. Ringel, "Oral tactile experience," Arch. Oral Biol., vol. 10, pp. 691-705, 1965.

[17] R. L. Ringel and S. J. Ewanowshi, "Oral perception: 1. Two-point discrimination," J. Speech Hearing Res., vol. 8, pp. 389-398, 1965.

[18] P. Bach-y-Rita, K. A. Kaczmarek, M. E. Tyler, and J. Garcia-Lara, "Form perception with a 49-point electrotactile stimulus array on the tongue: A technical note," J. Rehabil. Res. Develop., vol. 35, pp. 427-430, 1998.

[19] P. A. Dagenais, "Electropalatography in the treatment of articulation/phonological disorders," J. Commun. Disorders, vol. 28, pp. 303-329, 1995.

[20] C. Salem and S. Zhai, "An isometric tongue pointing device," presented at the CHI Conf. Human Factors Computer Syst., Atlanta, GA, 1997.

[21] C. Lau and S. Oleary, "Comparison of computer-interface devices for persons with severe physical-disabilities," Amer. J. Occupational Therapy, vol. 47, no. 11, pp. 1022-1030, 1993.

[22] W. Nutt, C. Arlanch, S. Nigg, and G. Staufert, "Tongue-mouse for quadriplegics," J. Micromech. Microeng., vol. 8, no. 2, pp. 155-157, 1998.

[23] H. Tang and D. J. Beebe, "Design and microfabrication of a flexible oral electrotactile display," J. Microelectromechanical Syst., vol. 12, no. 1, pp. 29-36, 2003.

[24] R. L. Ringel and S. J. Ewanowshi, "Oral perception: 1. Two-point discrimination," J. Speech Hearing Res., vol. 8, pp. 389-398, 1965.

[25] R. M. Strong and D. E. Troxel, "An electrotactile display," IEEE Trans. Man-Machine Syst., vol. MMS-11, no. 1, pp. 72-79, 1970.

[26] H. Tang and D. J. Beebe, "A microfabricated electrostatic haptic display for persons with visual impairments," IEEE Trans. Rehabil. Eng., vol. 6, no. 3, pp. 241-248, Sep. 1998.

[27] N. A. Macmillan and C. D. Creelman, Detection Theory: A User's Guide. New York: Cambridge Univ. Press, 1991. 
Hui Tang (S'97-M'00) received the B.S. and M.S. degrees in electrical engineering from the University of Science \& Technology of China (USTC), Anhui, China, in 1987 and 1990, respectively, the M.S. degree in biomedical engineering from Louisiana Tech University, Ruston, in 1996, and the Ph.D. degree in electrical engineering from University of Illinois, Urbana-Champaign (UIUC), in 2000

He has been an Assistant Professor in the Department of Electrical and Computer Engineering, University of Missouri-Columbia since 2000. His research interests are in the interdisciplinary area of biomedical instrumentation and BioMEMS, focusing on innovative microtechnologies with significant impact in biomedical and rehabilitation fields. His ongoing research projects include wearable electrotactile displays (modeling, microfabrication, and new tactile coding methods), microinstruments for sensing and analysis of single cells, and nonoptical MEMS structures for nanoscale biological sensing. He has been teaching undergraduate and graduate courses on micromachined transducers, biomedical instrumentation, digital logic, and microcontroller interfacing. He also serves as a manuscript reviewer for international journals on MEMS and sensor technology.
David J. Beebe (S'89-M'95) received the B.S., M.S., and Ph.D. degrees in electrical engineering from the University of Wisconsin-Madison, in 1987, 1990, and 1994, respectively.

$\mathrm{He}$ is a Professor in the Department of Biomedical Engineering, the University of Wisconsin-Madison. He is also a member of the University of Wisconsin Comprehensive Cancer Center, Stem Cell Program, Materials Science Program, Biotechnology Training Program, Genomic Sciences Training Program, and serves on the steering committee of the Stem Cell Training Program. From 1996 to 1999, he was an Assistant Professor in the Department of Electrical and Computer Engineering and an Assistant Research Professor in the Beckman Institute for Advanced Science and Technology, the University of Illinois, Urbana-Champaign. From 1994 to 1996, he was an Assistant Professor at Louisiana Tech University, Ruston. He has also served as an Associate Editor for the Journal of Biomechanical Engineering and is currently on the editorial board of Lab-on-a-Chip. Past research topics have included development of nontraditional autonomous microfluidic devices and systems, and the study of cell and embryo development in microenvironments. His current interests center around understanding the role stem/progenitor cells play in the development of the mammary gland. He is currently in the midst of a retraining effort in cancer biology via a five year National Institutes of Health K25 award.

Dr. Beebe is the recipient of the IEEE EMBS Early Career Achievement Award and the Romnes Award at UW-Madison. He is an Associate Editor for IEEE JOURNAL OF MicROELECTROMECHANICAL SYSTEMS. 